\title{
Determination of the Vesicular Systems Parameters: Parallel Implementation and Analysis of the PTNS Vesicle Structure
}

\author{
Maxim Bashashin ${ }^{1,2, \star}$, Elena Zemlyanaya ${ }^{1,2}$, Evgeniya Zhabitskaya ${ }^{1,2}$, Mikhail Kiselev ${ }^{1,2}$, and \\ Tatyana Sapozhnikova ${ }^{1}$ \\ ${ }^{1}$ Joint Institute for Nuclear Research, Joliot-Curie str. 6, 141980 Dubna, Russia; \\ ${ }^{2}$ Dubna State University, University str. 19, 141980 Dubna, Russia
}

\begin{abstract}
Parameters of the phospholipid vesicular systems are determined by means of minimization of the discrepancy between experimental data on the small angle scattering intensity and the numerical results within the frame of the separated form factors method. Effectiveness of parallel implementation is tested at the cluster HybriLIT. Results of numerical analysis of small angle neutron scattering data on polydispersed population of unilamellar vesicles of phospholipid transport nanosystem are presented.
\end{abstract}

\section{Introduction}

Phospholipid vesicles (liposomes, nanospheres) have a wide range of applications in modern biochemistry and pharmacology. One of the important trends is the development of the drug delivery systems on the basis of phospholipds [1,2]. The small angle scattering of neutrons (SANS) and of Xrays (SAXS) are known as powerful tools for the nanosystems structure investigation. SANS and SAXS measurements are well suited for the analysis of the structure of the polydispersed populations of unilamellar vesicles (ULVs) of phospholipids in water solutions of disaccharides [3-6]. For this analysis, we utilize the separated form factors method (SFF) [6-8]. Parameters of ULVs are adjusted by means of the weighted least square method

$$
\chi^{2}=\frac{1}{N-k} \sum_{j=1}^{N}\left(\frac{I^{\text {theor }}\left(q_{j}\right)-I^{\text {exper }}\left(q_{j}\right)}{\delta^{\operatorname{exper}}\left(q_{j}\right)}\right)^{2},
$$

where $I^{\text {theor }}\left(q_{j}\right)$ and $I^{\text {exper }}\left(q_{j}\right)$ are, respectively, theoretical and experimental small angle intensity values at the points $q_{j}$ of the scattering vector, $N$ is the number of experimental points, $k$ is the number of parameters to be fitted, $\delta$ are the experimental errors.

In this contribution, we discuss the effectiveness of parallel implementation of the SFF-parameters fitting procedure. Also, results of the SFF analysis of SANS data on polydispersed population of ULVs of phospholipid transport nanosystem (PTNS) are presented.

\section{Separated form factors method}

The SFF method for analysis of SANS data is determined by the following formulas (details are given in $[3,4,6-8])$. The expression of the intensity $I(q)$ has the following form:

\footnotetext{
^e-mail: bashashinmv@jinr.ru
} 


$$
I(q)=I_{m}(q)+\frac{1}{2} \Delta^{2} \frac{d^{2} I_{m}(q)}{d q^{2}}+I_{B}, \quad I_{m}(q)=\left[\int_{R_{\min }}^{R_{\max }} I_{\mathrm{SFF}}(q, R) G(R, \bar{R}) d R\right] \times\left[\int_{R_{\min }}^{R_{\max }} G(R, \bar{R}) d R\right]^{-1}
$$

where $q$ is the scattering vector, $I_{B}$ characterizes the incoherent background, $\Delta^{2}$ is the second momentum of spectrometer resolution function. $I_{m}$ is the ULV system SANS intensity with average radius $\bar{R}$. Polydispersity $\sigma$ is accounted for by the Schulz distribution $G(R, \bar{R})$ with coefficient $m$ :

$$
G(R, \bar{R})=\frac{R^{m}}{m !}\left(\frac{m+1}{\bar{R}}\right)^{m+1} \exp \left[-\frac{(m+1) R}{\bar{R}}\right], \quad \sigma=\frac{1}{\sqrt{m+1}} .
$$

$I_{\mathrm{SFF}}$ is the intensity of monodispersed system of ULVs:

$$
I_{\mathrm{SFF}}(q)=n I_{o} F_{s}(q) F_{b}(q) S(q), \quad F_{s}(q)=\left(4 \pi R^{2} \frac{\sin (q R)}{q R}\right)^{2}, \quad F_{b}(q)=\left(\int_{d / 2}^{+d / 2} \rho_{c}(x) \cos (q x) d x\right)^{2},
$$

where $I_{o}$ is the intensity of the incident beam, $n$ is a number of vesicles in $\mathrm{cm}^{3}, F_{s}$ is the form factor of the spherical surface with radius $R, F_{b}$ is the form factor of the symmetric lipid bilayer of thickness $d, \rho_{c}(x)$ is the contrast between the scattering length density of the lipid bilayer and the density of the solvent, $S(q) \sim 1$ is a structural factor. Here, we use the hydrophilic-hydrophobic model $(\mathrm{HH})$ of the density distribution across bilayer suggested in [8] and shown in Fig. 1(a).

\section{Parallel implementation}

In our calculations, we employ both global and local minimization for the ULVs SFF-parameters adjustment. The global minimization is based on the asynchronous differential evolution algorithm (ADE). The ADE method incorporates the classical differential evolutions algorithm into a parallel steady-state strategy. ADE minimiser with adaptive correlation matrix automatically adapts to the landscape of the optimized objective function, see [11] and references therein for details. ADE approach was successfully employed for the SFF analysis of SAXS data [7, 12]. High effectiveness of parallel implementation of SFF-ADE calculations is demonstrated in [12].

On the other hand, in many cases, the local minimization is also effective. The iterative minimization procedure implemented in the code DFUMIL ${ }^{1}$ and FUMILIM [9], provides quick convergence to the minimum of the $\chi^{2}$ expression (1), see [10] and references therein for details. In the parallel MPI-version of this method (PFUMILI ${ }^{2}$ ), the parallelism is based on a distribution of the set of experimental points between parallel MPI-processes, with the collection of intermediate results to the master process at each iteration. Because of the intensive interaction between the MPI-processes, significant acceleration of calculations on multi-processor systems (as we observe in the case of ADE calculations) is not available. Nevertheless, in case of relatively small number of parallel processes, one expects a noticeable decrease of computer time.

In order to test the effectivenes of the PFUMILI-based parallel implementation of the SFFparameters fitting procedure, we have made methodical calculations for two sets of SANS experimental data on a polydispersed ULVs population of dimirostoilphosphatidilholin (DMPC) in $\mathrm{D}_{2} \mathrm{O}$.

\footnotetext{
${ }^{1}$ https://wwwinfo.jinr.ru/programs/jinrlib/d510

${ }^{2}$ https://wwwinfo.jinr.ru/programs/jinrlib/pfumili
} 

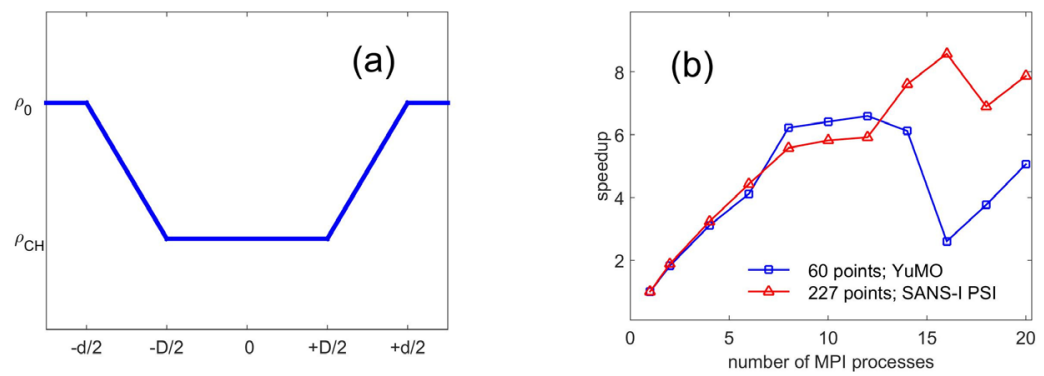

Figure 1. (a) HH-model of the the scattering length density across bilayer: $d$ is the bilayer thickness, $D$ is the thickness of hydrophobic region, $\rho_{C H}=-0.36 \cdot 10^{10}$ is the hydrocarbon chains scattering length density, $\rho_{0}$ is the solvent density. (b) Speedup of PFUMILI-SFF calculations versus the number of MPI-processes in case of analysis of the 60-point YuMO spectrum and the 227-points SANS-I spectrum.
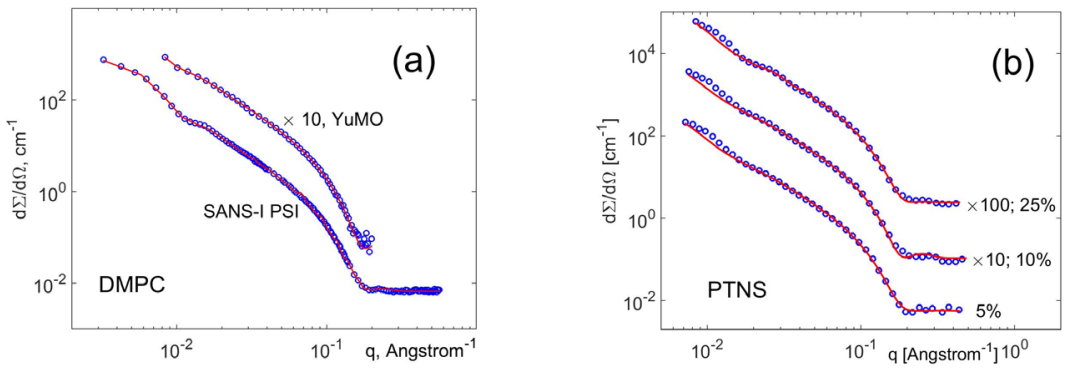

Figure 2. (a) Theoretical and experimental spectra of the polydispersed DMPC ULV system in $\mathrm{D}_{2} 0$. SANS data have been obtained at spectrometers YuMO (Dubna) and SANS-I PSI (Villingen). (b) Theoretical and experimental spectra of polydispersed population of PTNS ULVs in the heavy water solvent of maltose with different concentrations.

The first spectrum with 60 experimental points was measured at the YuMO spectrometer (Dubna). The second one (227 experimental points) was obtained at the SANS-I PSI facility (Willingen). The speedup of the calculations versus the number of MPI-processes is shown in Fig. 1(b). The maximal speedup is about 6.6 times for the 60-point YuMO spectrum (12 MPI-processes) and about 8.6 time for the 227-points SANS-I spectrum (16 MPI-processes). Obviously the parallel implementation efficiency should be increased as the number of experimental points is growing.

The values of basic adjusted parameters (see Table 1) are in agreement with the results in [8]. Fig. 2(a) demonstrates the respective experimental and theoretical curves.

Table 1. Basic parameters of polydispersed populations of DMPC and PTNS ULVs. Adjusted parameters: the average radius $\bar{R}$, the polydispersity coefficient $m$, the bilayer thickness $d$, the hydrophobic region thickness $D$.

\begin{tabular}{llllllll}
\hline Experiment & Maltose & $\rho_{0}, \mathrm{~cm}^{-2}$ & $\bar{R}, \AA$ & $m$ & $d, \AA$ & $\mathrm{D}, \AA$ & $\chi^{2}$ \\
\hline YuMO; DMPC & $0 \%$ & $6.37 \cdot 10^{10}$ & $277.0 \pm 5.0$ & $9.8 \pm 0.7$ & $49.0 \pm 2.0$ & $20.0 \pm 3.0$ & 1.1 \\
SANS-I; DMPC & $0 \%$ & $6.37 \cdot 10^{10}$ & $275.6 \pm 0.5$ & $12.6 \pm 0.3$ & $47.8 \pm 0.2$ & $20.5 \pm 0.4$ & 1.7 \\
\hline YuMO; PTNS & $5 \%$ & $6.16 \cdot 10^{10}$ & $190.4 \pm 4$ & $4.7 \pm 0.5$ & $50.6 \pm 0.2$ & $2.0 \pm 0.2$ & 7.0 \\
YuMO; PTNS & $10 \%$ & $5.95 \cdot 10^{10}$ & $200.3 \pm 2.1$ & $4.8 \pm 0.5$ & $51.3 \pm 1.9$ & $2.0 \pm 0.9$ & 8.0 \\
YuMO; PTNS & $25 \%$ & $5.33 \cdot 10^{10}$ & $192.1 \pm 1.4$ & $7.6 \pm 0.7$ & $47.7 \pm 4.7$ & $11.9 \pm 2.0$ & 8.2 \\
\hline
\end{tabular}




\section{Analysis of PTNS ULVs structure}

The phospholipid transport nanosystem (PTNS) with extremely small ULVs size has been obtained on the basis of the soybean phosphatidylcholine in the Orekhovich Research Institute of Biomedical Chemistry (Moscow, Russia). Incorporation of drugs into PTNS ULVs is found to increase sufficiently the therapeutic effectiveness. The structure of PTNS ULVs was analysed in $[12,13]$ on the basis of SAXS data collected at the Kurchatov Synchrotron Radiation Source of the NRC "Kurchatov Institute" (Moscow, Russia). Here, we present first estimations of basic parameters of PTNS ULVs in the heavy water solvent of maltose on the basis of the HH-SFF analysis of SANS data collected at YuMO spectrometer. Fig. 2(b) demonstrates a reasonable agreement of the SFF calculations with experimental data. Values of basic parameters of PTNS system are given in Table 1. The results of both local and global minimization are in very good agreement.

From Table 1 it follows that the average radius $\bar{R}$ of PTNS ULVs is 1.5 times smaller then the radius of "classical" DMPC ULVs. Note also the weak dependence of $\bar{R}$ on maltose concentration. The PTNS and DMPC bilayer thicknesses $d$ are close to each other. At 5\% and 10\% concentration we obtained extremely small values of the hydrophobic region $D$ in bilayer. This requires further analysis and explanation.

\section{Summary}

We have found that the PFUMILI-based parallel implementation of the SFF-parameters fitting procedure provides the 6-9 times speedup in comparison with the sequential calculations. First estimations of the PTNS ULVs structure within the frame of the HH-SFF analysis of SANS data collected at YuMO spectrometer have been done. Parallel versions of both ADE-based and PFUMILI-based fitting procedures are expected to accelerate the further study of PTNS ULVs structure on the basis of the SFF analysis of SANS and SAXS data.

\section{Acknowledgement}

The work has been performed under the grant of Russian Scientific Foundation (project No 14-1200516).

\section{References}

[1] S. Das and A. Chaudhury, AAPS PharmSci. Tech. 12, 62-76 (2011)

[2] S. Martins, B. Sarmento, D.C. Ferreira, E.B. Souto, Int. J. Nanomedicine 2, 595-607 (2007)

[3] M.A. Kiselev et al, Nucl. Inst. \& Method A 470, 409-416 (2001)

[4] M. Kiselev et al, J. Alloys and Compounds 328, 71-76 (2001)

[5] M.A. Kiselev et al, Chemistry and Physics of Lipids 123, 31-44 (2003)

[6] M.A. Kiselev et al, Colloids and Surfaces A 256 1-7 (2005)

[7] M.A. Kiselev, E.V. Zemlyanaya, E.I. Zhabitskaya, V.L. Aksenov, Crystallography Reports 60, 143-147 (2015)

[8] M.A. Kiselev, E.V. Zemlyanaya, V.K. Aswal, R. Neubert, European Biophys. J 35, 477-493 (2006)

[9] I.M. Sitnik, Comput. Phys. Comm. 209, 199-2004 (2016)

[10] V.S. Kurbatov and I.N. Silin, Nucl. Instr. \& Meth. A 345, 346-350 (1994)

[11] E.I. Zhabitskaya and M.V. Zhabitsky, Proc. 15th Ann. Conf. on Genetic and Evolutionary Comput., (USA, N.Y., 2013) 455-462

[12] E. Zhabitskaya, E. Zemlyanaya, M. Kiselev, A. Gruzinov, EPJ Web of Conf. 108, 02047 (2016)

[13] M.A. Kiselev et al, J. Pharmaceutical and Biomedical Analysis 114, 288-291 (2015) 